\title{
On the convergence of the combination technique
}

\author{
Michael Griebel and Helmut Harbrecht
}

\begin{abstract}
Sparse tensor product spaces provide an efficient tool to discretize higher dimensional operator equations. The direct Galerkin method in such ansatz spaces may employ hierarchical bases, interpolets, wavelets or multilevel frames. Besides, an alternative approach is provided by the so-called combination technique. It properly combines the Galerkin solutions of the underlying problem on certain full (but small) tensor product spaces. So far, however, the combination technique has been analyzed only for special model problems. In the present paper, we provide now the analysis of the combination technique for quite general operator equations in sparse tensor product spaces. We prove that the combination technique produces the same order of convergence as the Galerkin approximation with respect to the sparse tensor product space. Furthermore, the order of the cost complexity is the same as for the Galerkin approach in the sparse tensor product space. Our theoretical findings are validated by numerical experiments.
\end{abstract}

\section{Introduction}

The discretization in sparse tensor product spaces yields efficient numerical methods to solve higher dimensional operator equations. Nevertheless, a Galerkin discretization in these sparse tensor product spaces requires hierarchical bases, interpolets, wavelets, multilevel frames, or other types of multilevel systems $[9,12,18]$ which make a direct Galerkin discretization in sparse tensor product spaces quite involved and cumbersome in practical applications. To avoid these issues of the Galerkin dis-

Michael Griebel

Institut für Numerische Simulation, Universität Bonn, Wegelerstr. 6, 53115 Bonn, Germany,

e-mail: griebel@ins.uni-bonn.de

Helmut Harbrecht

Mathematisches Institut, Universität Basel, Rheinsprung 21, 4051 Basel, Switzerland,

e-mail: helmut.harbrecht@unibas.ch 
cretization, the combination technique has been introduced in [14]. There, only the Galerkin discretizations and solutions in appropriately chosen, full, but small, tensor product spaces need to be computed and combined.

In $[8,16,19]$, it has been shown that, in the special case of operator equations which involve a tensor product operator, the approximation produced by the combination technique indeed coincides exactly with the Galerkin solution in the sparse tensor product space. However, for non-tensor product operators, this is no longer the case. Nevertheless, it is observed in practice that the approximation error is of the same order. But theoretical convergence results are only available for specific applications, see for example [3, 14, 21, 22, 23, 25]. Moreover, a general proof of convergence is so far still missing for the combination technique.

In the present paper, we prove optimal convergence rates of the combination technique for elliptic operators acting on arbitrary Gelfant triples. The convergence analysis is based on two compact lemmas (Lemma 1 and Lemma 2) which have basically been proven in [22, 25]. In contrast to these papers, besides considering abstract Gelfant triples, we deal here with the combination technique for the so-called generalized sparse tensor product spaces which have been introduced in [10]. Lemma 1 involves a special stability condition for the Galerkin projection (cf. (18)) which, however, holds for certain regularity assumptions on the operator under consideration (see Remark 1).

To keep the notation and the proofs simple, we restrict ourselves to the case of operator equations which are defined on a two-fold product domain $\Omega_{1} \times \Omega_{2}$. However, we allow the domains $\Omega_{1} \subset \mathbb{R}^{n_{1}}$ and $\Omega_{2} \subset \mathbb{R}^{n_{2}}$ to be of different spatial dimensions. Our proofs can be generalized without further difficulties to arbitrary $L$-fold product domains $\Omega_{1} \times \Omega_{2} \times \cdots \times \Omega_{L}$ by employing the techniques from [11] and [25].

The remainder of this paper is organized as follows. We first present the operator equations under consideration in Section 2. Then, in Section 3, we specify the requirements of the multiscale hierarchies on each individual subdomain. In Section 4, we define the generalized sparse tensor product spaces and recall their basic properties. The combination technique is introduced in Section 5 and its convergence is proven in Section 6. Section 7 is dedicated to numerical experiments. They are in good agreement with the presented theory. Finally, in Section 8, we give some concluding remarks.

Throughout this paper, the notion "essential" in the context of complexity estimates means "up to logarithmic terms". Moreover, to avoid the repeated use of generic but unspecified constants, we signify by $C \lesssim D$ that $C$ is bounded by a multiple of $D$ independently of parameters which $C$ and $D$ may depend on. Obviously, $C \gtrsim D$ is defined as $D \lesssim C$, and $C \sim D$ as $C \lesssim D$ and $C \gtrsim D$. 


\section{Operator equations}

We consider two sufficiently smooth, bounded domains $\Omega_{1} \in \mathbb{R}^{n_{1}}$ and $\Omega_{2} \in \mathbb{R}^{n_{2}}$, where $n_{1}, n_{2} \in \mathbb{N}$. Moreover, on the product domain $\Omega_{1} \times \Omega_{2}$, let the Hilbert space $\mathscr{H}$ be given such that

$$
\mathscr{H} \subset L^{2}\left(\Omega_{1} \times \Omega_{2}\right) \subset \mathscr{H}^{\prime}
$$

forms a Gelfand triple. Thus, the inner product

$$
(u, v)_{L^{2}\left(\Omega_{1} \times \Omega_{2}\right)}:=\int_{\Omega_{1}} \int_{\Omega_{2}} u(\mathbf{x}, \mathbf{y}) v(\mathbf{x}, \mathbf{y}) \mathrm{d} \mathbf{x} \mathrm{d} \mathbf{y}
$$

in $L^{2}\left(\Omega_{1} \times \Omega_{2}\right)$ can continuously be extended to $\mathscr{H} \times \mathscr{H}^{\prime}$. For sake of simplicity of presentation, we write $(u, v)_{L^{2}\left(\Omega_{1} \times \Omega_{2}\right)}$ also in the case $u \in \mathscr{H}$ and $v \in \mathscr{H}^{\prime}$.

Now, let $A: \mathscr{H} \rightarrow \mathscr{H}^{\prime}$ denote a differential or pseudo-differential operator. It is assumed that it maps the Hilbert space $\mathscr{H}$ continuously and bijectively onto its dual $\mathscr{H}^{\prime}$, i.e.,

$$
\|A u\|_{\mathscr{H}^{\prime}} \sim\|u\|_{\mathscr{H}} \text { for all } u \in \mathscr{H} .
$$

The Hilbert space $\mathscr{H}$ is thus the energy space of the operator under consideration. For the sake of simplicity, we further assume that $A$ is $\mathscr{H}$-elliptic. Consequently, the resulting bilinear form

$$
a(u, v):=(A u, v)_{L^{2}\left(\Omega_{1} \times \Omega_{2}\right)}: \mathscr{H} \times \mathscr{H} \rightarrow \mathbb{R}
$$

is continuous

$$
a(u, v) \lesssim\|u\|_{\mathscr{H}}\|v\|_{\mathscr{H}} \text { for all } u, v \in \mathscr{H}
$$

and elliptic

$$
a(u, u) \gtrsim\|u\|_{\mathscr{H}}^{2} \text { for all } u \in \mathscr{H} .
$$

In the following, for given $f \in \mathscr{H}^{\prime}$, we want to efficiently solve the operator equation $A u=f$ or, equivalently, the variational formulation:

$$
\text { find } u \in \mathscr{H} \text { such that } a(u, v)=(f, v)_{L^{2}\left(\Omega_{1} \times \Omega_{2}\right)} \text { for all } v \in \mathscr{H} \text {. }
$$

Of course, since we like to focus on conformal Galerkin discretizations, we should tacitly assume that, for all $j_{1}, j_{2} \geq 0$, the tensor product $V_{j_{1}}^{(1)} \otimes V_{j_{2}}^{(2)}$ of the ansatz spaces $V_{j_{1}}^{(1)}$ and $V_{j_{2}}^{(2)}$ is contained in the energy space $\mathscr{H}$. Moreover, for the solution $u \in \mathscr{H}$ of (1), we will need a stronger regularity to hold for obtaining decent convergence rates. Therefore, for $s_{1}, s_{2} \geq 0$, we introduce the following Sobolev spaces of dominant mixed derivatives with respect to the underlying space $\mathscr{H}$

$$
\mathscr{H}_{\text {mix }}^{s_{1}, s_{2}}:=\left\{f \in \mathscr{H}:\left\|\frac{\partial^{\alpha+\beta}}{\partial_{\mathbf{x}}^{\alpha} \partial_{\mathbf{y}}^{\beta}} f\right\|_{\mathscr{H}}<\infty \text { for all }|\alpha| \leq s_{1} \text { and }|\beta| \leq s_{2}\right\} .
$$

We shall illustrate our setting by the following specific examples. 
Example 1. A first simple example is the operator $A: L^{2}\left(\Omega_{1} \times \Omega_{2}\right) \rightarrow L^{2}\left(\Omega_{1} \times \Omega_{2}\right)$ which underlies the bilinear form

$$
a(u, v)=\int_{\Omega_{1}} \int_{\Omega_{2}} \alpha(\mathbf{x}, \mathbf{y}) u(\mathbf{x}, \mathbf{y}) v(\mathbf{x}, \mathbf{y}) \mathrm{d} \mathbf{x} \mathrm{d} \mathbf{y},
$$

where the coefficient function $\alpha$ satisfies

$$
0<\underline{\alpha} \leq \alpha(\mathbf{x}, \mathbf{y}) \leq \bar{\alpha} \text { for all }(\mathbf{x}, \mathbf{y}) \in \Omega_{1} \times \Omega_{2}
$$

Here, it holds $\mathscr{H}=L^{2}\left(\Omega_{1} \times \Omega_{2}\right)$. Moreover, our spaces $\mathscr{H}_{\text {mix }}^{s_{1}, s_{2}}$ of assumed stronger regularity coincide with the standard Sobolev spaces of dominant mixed derivatives, i.e.,

$$
\mathscr{H}_{\text {mix }}^{s_{1}, s_{2}}=H_{\text {mix }}^{s_{1}, s_{2}}\left(\Omega_{1} \times \Omega_{2}\right):=H^{s_{1}}\left(\Omega_{1}\right) \otimes H^{s_{2}}\left(\Omega_{2}\right) .
$$

Example 2. Stationary heat conduction in the product domain $\Omega_{1} \times \Omega_{2}$ yields the bilinear form

$$
a(u, v)=\int_{\Omega_{1}} \int_{\Omega_{2}} \alpha(\mathbf{x}, \mathbf{y})\left\{\nabla_{\mathbf{x}} u(\mathbf{x}, \mathbf{y}) \nabla_{\mathbf{x}} v(\mathbf{x}, \mathbf{y})+\nabla_{\mathbf{y}} u(\mathbf{x}, \mathbf{y}) \nabla_{\mathbf{y}} v(\mathbf{x}, \mathbf{y})\right\} \mathrm{d} \mathbf{x} \mathrm{d} \mathbf{y} .
$$

If the coefficient $\alpha$ satisfies (2), then the associated operator $A$ is known to be continuous and elliptic with respect to the space $\mathscr{H}=H_{0}^{1}\left(\Omega_{1} \times \Omega_{2}\right)$. Moreover, our spaces $\mathscr{H}_{\text {mix }}^{s_{1}, s_{2}}$ of assumed stronger regularity now coincide with $\mathscr{H}_{\text {mix }}^{s_{1}, s_{2}}=$ $H_{0}^{1}\left(\Omega_{1} \times \Omega_{2}\right) \cap H_{\text {mix }}^{s_{1}+1, s_{2}}\left(\Omega_{1} \times \Omega_{2}\right) \cap H_{\text {mix }}^{s_{1}, s_{2}+1}\left(\Omega_{1} \times \Omega_{2}\right)$.

Example 3. Another example appears in two-scale homogenization. Unfolding ([4]) gives raise to the product of the macroscopic physical domain $\Omega_{1}$ and the periodic microscopic domain $\Omega_{2}$ of the cell problem, see [20]. Then, for the first order corrector, one arrives at the bilinear form

$$
a(u, v)=\int_{\Omega_{1}} \int_{\Omega_{2}} \alpha(\mathbf{x}, \mathbf{y}) \nabla_{\mathbf{y}} u(\mathbf{x}, \mathbf{y}) \nabla_{\mathbf{y}} v(\mathbf{x}, \mathbf{y}) \mathrm{d} \mathbf{x} \mathrm{d} \mathbf{y} .
$$

The underlying operator $A$ is continuous and elliptic as a operator in the related energy space $\mathscr{H}=L^{2}\left(\Omega_{1}\right) \otimes H_{0}^{1}\left(\Omega_{2}\right)$ provided that the coefficient $\alpha$ satisfies again (2). Furthermore, our spaces $\mathscr{H}_{m i x}^{s_{1}, s_{2}}$ of assumed stronger regularity coincide with $\mathscr{H}_{\text {mix }}^{s_{1}, s_{2}}=\left(L^{2}\left(\Omega_{1}\right) \otimes H_{0}^{1}\left(\Omega_{2}\right)\right) \cap H_{\text {mix }}^{s_{1}, s_{2}+1}\left(\Omega_{1} \times \Omega_{2}\right)$.

\section{Approximation on the individual subdomains}

On each domain $\Omega_{i}$, we consider a nested sequence

$$
V_{0}^{(i)} \subset V_{1}^{(i)} \subset \cdots \subset V_{j}^{(i)} \subset \cdots \subset L^{2}\left(\Omega_{i}\right)
$$

of finite dimensional spaces 


$$
V_{j}^{(i)}=\operatorname{span}\left\{\varphi_{j, k}^{(i)}: k \in \Delta_{j}^{(i)}\right\}
$$

(the set $\Delta_{j}^{(i)}$ denotes a suitable index set) of piecewise polynomial ansatz functions, such that $\operatorname{dim} V_{j}^{(i)} \sim 2^{j n_{i}}$ and

$$
L^{2}\left(\Omega_{i}\right)=\overline{\bigcup_{j \in \mathbb{N}_{0}} V_{j}^{(i)}}
$$

We will use the spaces $V_{j}^{(i)}$ for the approximation of functions. To this end, we assume that the approximation property

$$
\inf _{v_{j} \in V_{j}^{(i)}}\left\|u-v_{j}\right\|_{H^{q}\left(\Omega_{i}\right)} \lesssim h_{j}^{s-q}\|u\|_{H^{s}\left(\Omega_{i}\right)}, \quad u \in H^{s}\left(\Omega_{i}\right),
$$

holds for $q<\gamma_{i}, q \leq s \leq r_{i}$ uniformly in $j$. Here we set $h_{j}:=2^{-j}$, i.e., $h_{j}$ corresponds to the width of the mesh associated with the subspace $V_{j}^{(i)}$ on $\Omega_{i}$. The parameter $\gamma_{i}>0$ refers to the regularity of the functions which are contained in $V_{j}^{(i)}$, i.e.,

$$
\gamma_{i}:=\sup \left\{s \in \mathbb{R}: V_{j}^{(i)} \subset H^{s}\left(\Omega_{i}\right)\right\} .
$$

The integer $r_{i}>0$ refers to the polynomial exactness, that is the maximal order of polynomials which are locally contained in the space $V_{j}^{(i)}$.

Now, let $Q_{j}^{(i)}: L^{2}\left(\Omega_{i}\right) \rightarrow V_{j}^{(i)}$ denote the $L^{2}\left(\Omega_{i}\right)$-orthogonal projection onto the finite element space $V_{j}^{(i)}$. Due to the orthogonality, we have $\left(Q_{j}^{(i)}\right)^{\star}=Q_{j}^{(i)}$. Moreover, our regularity assumptions on the ansatz spaces $V_{j}^{(i)}$ imply the continuity of the related projections relative to the Sobolev space $H^{q}\left(\Omega_{i}\right)$ for all $|q|<\gamma_{i}$, i.e., it holds

$$
\left\|Q_{j}^{(i)} u\right\|_{H^{q}\left(\Omega_{i}\right)} \lesssim\|u\|_{H^{q}\left(\Omega_{i}\right)}, \quad|q|<\gamma_{i},
$$

uniformly in $j \geq 0$ provided that $u \in H^{q}\left(\Omega_{i}\right)$.

By setting $Q_{-1}^{(i)}:=0$, we can define for all $j \geq 0$ the complementary spaces

$$
W_{j}^{(i)}:=\left(Q_{j}^{(i)}-Q_{j-1}^{(i)}\right) L^{2}\left(\Omega_{i}\right) \subset V_{j}^{(i)} .
$$

They satisfy

$$
V_{j}^{(i)}=V_{j-1}^{(i)} \oplus W_{j}^{(i)}, \quad V_{j-1}^{(i)} \cap W_{j}^{(i)}=\{0\},
$$

which recursively yields

$$
V_{J}^{(i)}=\bigoplus_{j=0}^{J} W_{j}^{(i)}
$$

A given function $f \in H^{q}\left(\Omega_{i}\right)$, where $|q|<\gamma_{i}$, admits the unique multiscale decomposition 


$$
f=\sum_{j=0}^{\infty} f_{j} \text { with } f_{j}:=\left(Q_{j}^{(i)}-Q_{j-1}^{(i)}\right) f \in W_{j}^{(i)} .
$$

One now has the well-known norm equivalence

$$
\|f\|_{H^{q}\left(\Omega_{i}\right)}^{2} \sim \sum_{j=0}^{\infty} 2^{2 j q}\left\|\left(Q_{j}^{(i)}-Q_{j-1}^{(i)}\right) f\right\|_{L^{2}\left(\Omega_{i}\right)}^{2}, \quad|q|<\gamma_{i},
$$

see [5]. Finally, for any $f \in H^{s}\left(\Omega_{i}\right)$ and $|q|<\gamma_{i}$, the approximation property (4) induces the estimate

$$
\left\|\left(Q_{j}^{(i)}-Q_{j-1}^{(i)}\right) f\right\|_{H^{q}\left(\Omega_{i}\right)} \lesssim 2^{-j(s-q)}\|f\|_{H^{s}\left(\Omega_{i}\right)}, \quad q<s \leq r_{i} .
$$

\section{Generalized sparse tensor product spaces}

The canonical approximation method in the Hilbert space $\mathscr{H}$ is the approximation in full tensor product spaces ${ }^{1}$

$$
V_{J / \sigma}^{(1)} \otimes V_{J \sigma}^{(2)}=\bigoplus_{\substack{j_{1} \sigma \leq J \\ j_{2} / \sigma \leq J}} W_{j_{1}}^{(1)} \otimes W_{j_{2}}^{(2)} .
$$

Here, $\sigma>0$ is a given parameter which can be tuned to optimize the cost complexity. There are $2^{J n_{1} / \sigma} \cdot 2^{J n_{2} \sigma}$ degrees of freedom in the space $V_{J / \sigma}^{(1)} \otimes V_{J \sigma}^{(2)}$. Moreover, for $f \in \mathscr{H}_{\text {mix }}^{s_{1}, 0}\left(\Omega_{1} \times \Omega_{2}\right) \cap \mathscr{H}_{\text {mix }}^{0, s_{2}}\left(\Omega_{1} \times \Omega_{2}\right)$ and $f_{J}:=\left(Q_{J / \sigma}^{(1)} \otimes Q_{J \sigma}^{(2)}\right) f \in V_{J / \sigma}^{(1)} \otimes V_{J \sigma}^{(2)}$, an error estimate of the type

$$
\left\|f-f_{J}\right\|_{\mathscr{H}} \lesssim 2^{-J \min \left\{s_{1} / \sigma, s_{2} \sigma\right\}}\|f\|_{\mathscr{H}_{\text {mix }}^{s_{1}, 0} \cap \mathscr{H}_{\text {mix }}^{0, s_{2}}}
$$

holds for all $0<s_{1} \leq p_{1}$ and $0<s_{2} \leq p_{2}$. Note that the upper bounds $p_{1}$ and $p_{2}$ are the largest values such that $\mathscr{H}_{\text {mix }}^{p_{1}, 0} \subset H_{\text {mix }}^{r_{1}, r_{2}}\left(\Omega_{1} \times \Omega_{2}\right)$ and $\mathscr{H}_{\text {mix }}^{0, p_{2}} \subset H_{\text {mix }}^{r_{1}, r_{2}}\left(\Omega_{1} \times \Omega_{2}\right)$, respectively.

Alternatively, based on the multiscale decompositions (6) on each individual subdomain, one can define the so-called generalized sparse tensor product space, see [1] and [10],

$$
\widehat{V}_{J}^{\sigma}:=\bigoplus_{j_{1} \sigma+j_{2} / \sigma \leq J} W_{j_{1}}^{(1)} \otimes W_{j_{2}}^{(2)}=\sum_{j_{1} \sigma+j_{2} / \sigma=J} V_{j_{1}}^{(1)} \otimes V_{j_{2}}^{(2)} .
$$

Thus, a function $f \in \mathscr{H}$ is represented by the Boolean sum

\footnotetext{
${ }^{1}$ Here and in the following, the summation limits are in general no natural numbers and must of course be rounded properly. We leave this to the reader to avoid cumbersome floor/ceil-notations.
} 


$$
\widehat{f}_{J}:=\sum_{j_{1} \sigma+j_{2} / \sigma \leq J} \Delta_{j_{1}, j_{2}}^{Q} f \in \widehat{V}_{J}^{\sigma}
$$

where, for all $j_{1}, j_{2} \geq 0$, the detail projections $\Delta_{j_{1}, j_{2}}^{Q}$ are given by

$$
\Delta_{j_{1}, j_{2}}^{Q}:=\left(Q_{j_{1}}^{(1)}-Q_{j_{1}-1}^{(1)}\right) \otimes\left(Q_{j_{2}}^{(2)}-Q_{j_{2}-1}^{(2)}\right)
$$

For further details on sparse grids we refer the reader to the survey [1] and the references therein.

The dimension of the generalized sparse tensor product space $\widehat{V}_{J}^{\sigma}$ is essentially equal to the dimension of the finest univariate finite element spaces which enter its construction, i.e., it is essentially equal to the value of $\max \left\{\operatorname{dim} V_{J / \sigma}^{(1)}, \operatorname{dim} V_{J \sigma}^{(2)}\right\}$. Nevertheless, by considering smoothness in terms of mixed Sobolev spaces, its approximation power is essentially the same as in the full tensor product space. To be precise, we have

Theorem 1 ([10]). The generalized sparse tensor product space $\widehat{V}_{J}^{\sigma}$ possesses

$$
\operatorname{dim} \widehat{V}_{J}^{\sigma} \sim \begin{cases}2^{J \max \left\{n_{1} / \sigma, n_{2} \sigma\right\}}, & \text { if } n_{1} / \sigma \neq n_{2} \sigma \\ 2^{J n_{1} / \sigma} J, & \text { if } n_{1} / \sigma=n_{2} \sigma\end{cases}
$$

degrees offreedom. Moreover, for a given function $f \in \mathscr{H}_{\text {mix }}^{s_{1}, s_{2}}$ and its $L^{2}$-orthonormal projection $\widehat{f}_{J} \in \widehat{V}_{J}^{\sigma}$, defined by (9), where $0<s_{1} \leq p_{1}$ and $0<s_{2} \leq p_{2}$, there holds the error estimate

$$
\left\|f-\widehat{f}_{J}\right\|_{\mathscr{H}} \lesssim \begin{cases}2^{-J \min \left\{s_{1} / \sigma, s_{2} \sigma\right\}}\|f\|_{\mathscr{H}_{\text {mix }}^{s_{1}, s_{2}}}, & \text { if } s_{1} / \sigma \neq s_{2} \sigma \\ 2^{-J s_{1} / \sigma} \sqrt{J}\|f\|_{\mathscr{H}_{\text {mix }}^{s_{1}, s_{2}},} & \text { if } s_{1} / \sigma=s_{2} \sigma .\end{cases}
$$

The optimal choice of the parameter $\sigma$ has been discussed in [10]. It turns out that the best cost complexity rate among all possible values of $s_{1}, s_{2}$ is obtained for the choice $\sigma=\sqrt{n_{1} / n_{2}}$. This choice induces an equilibration of the degrees of freedom in the extremal spaces $V_{J / \sigma}^{(1)}$ and $V_{J \sigma}^{(2)}$.

We shall consider the Galerkin discretization of (1) in the generalized sparse tensor product space $\widehat{V}_{J}^{\sigma}$, that is we want to

$$
\text { find } u_{J} \in \widehat{V}_{J}^{\sigma} \text { such that } a\left(u_{J}, v_{J}\right)=\left(f, v_{J}\right)_{L^{2}\left(\Omega_{1} \times \Omega_{2}\right)} \text { for all } v_{J} \in \widehat{V}_{J}^{\sigma} \text {. }
$$

In view of Theorem 1, we arrive at the following error estimate due to Céa's lemma.

Corollary 1. The Galerkin solution (11) satisfies the error estimate

$$
\left\|u-u_{J}\right\|_{\mathscr{H}} \lesssim\left\|u-\widehat{u}_{J}\right\|_{\mathscr{H}} \lesssim \begin{cases}2^{-J \min \left\{s_{1} / \sigma, s_{2} \sigma\right\}}\|u\|_{\mathscr{H}_{\text {mix }}^{s_{1}, s_{2}}}, & \text { if } s_{1} / \sigma \neq s_{2} \sigma \\ 2^{-J s_{1} / \sigma \sqrt{J}}\|u\|_{\mathscr{H}_{\text {mix }}^{s_{1}, s_{2}},} & \text { if } s_{1} / \sigma=s_{2} \sigma\end{cases}
$$

for all $0<s_{1} \leq p_{1}$ and $0<s_{2} \leq p_{2}$ provided that $u \in \mathscr{H}_{\text {mix }}^{s_{1}, s_{2}}$. 
Nevertheless, for the discretization of (11), hierarchical bases, interpolets, wavelets, multilevel frames, or other types of multilevel systems [1, 9, 12, 13, 17, 18, 24, 26] are required which make a direct Galerkin discretization in sparse tensor product spaces quite involved and cumbersome in practical applications.

\section{Combination technique}

The combination technique is a different approach for the discretization in sparse tensor product spaces. It avoids the explicit need of hierarchical bases, interpolets, wavelets or frames for the discretization of (11). In fact, one only has to compute the Galerkin solutions with respect to certain full tensor product spaces $V_{j_{1}}^{(1)} \otimes V_{j_{2}}^{(2)}$ and to appropriately combine them afterwords. The related Galerkin solutions $u_{j_{1}, j_{2}}$ are given by

$$
\begin{aligned}
& \text { find } u_{j_{1}, j_{2}} \in V_{j_{1}}^{(1)} \otimes V_{j_{2}}^{(2)} \text { such that } \\
& \qquad a\left(u_{j_{1}, j_{2}}, v_{j_{1}, j_{2}}\right)=\left(f, v_{j_{1}, j_{2}}\right)_{L^{2}\left(\Omega_{1} \times \Omega_{2}\right)} \text { for all } v_{j_{1}, j_{2}} \in V_{j_{1}}^{(1)} \otimes V_{j_{2}}^{(2)} .
\end{aligned}
$$

This introduces the Galerkin projection

$$
P_{j_{1}, j_{2}}: \mathscr{H} \rightarrow V_{j_{1}}^{(1)} \otimes V_{j_{2}}^{(2)}, \quad P_{j_{1}, j_{2}} u:=u_{j_{1}, j_{2}}
$$

which especially satisfies the Galerkin orthogonality

$$
a\left(u-P_{j_{1}, j_{2}} u, v_{j_{1}, j_{2}}\right)=0 \text { for all } v_{j_{1}, j_{2}} \in V_{j_{1}}^{(1)} \otimes V_{j_{2}}^{(2)} .
$$

The Galerkin projection $P_{j_{1}, j_{2}}$ is well defined for all $j_{1}, j_{2} \geq 0$ due to the ellipticity of the bilinear form $a(\cdot, \cdot)$. Moreover, as in (7), we conclude the error estimate

$$
\left\|u-P_{j_{1}, j_{2}} u\right\|_{\mathscr{H}} \lesssim\left\|u-\left(Q_{j_{1}}^{(1)} \otimes Q_{j_{2}}^{(2)}\right) u\right\|_{\mathscr{H}} \lesssim 2^{-\min \left\{j_{1} s_{1}, j_{2} s_{2}\right\}}\|u\|_{\mathscr{H}_{\text {mix }}^{s_{1}, 0} \cap \mathscr{H}_{\text {mix }}^{0, s_{2}}}
$$

for all $0<s_{1} \leq p_{1}$ and $0<s_{2} \leq p_{2}$ provided that $u \in \mathscr{H}_{\text {mix }}^{s_{1}, 0} \cap \mathscr{H}_{\text {mix }}^{0, s_{2}}$. In particular, for fixed $j_{1} \geq 0$ and $j_{2} \rightarrow \infty$, we obtain the Galerkin projection $P_{j_{1}, \infty}$ onto the space $V_{j_{1}, \infty}:=\left(Q_{j_{1}}^{(1)} \otimes I\right) \mathscr{H} \subset \mathscr{H}$. It satisfies the error estimate

$$
\left\|u-P_{j_{1}, \infty} u\right\|_{\mathscr{H}} \lesssim\left\|u-\left(Q_{j_{1}}^{(1)} \otimes I\right) u\right\|_{\mathscr{H}} \lesssim 2^{-j_{1} s_{1}}\|u\|_{\mathscr{H}_{\text {mix }}^{s_{1}, 0}}
$$

for all $0<s_{1} \leq p_{1}$. Likewise, for fixed $j_{2} \geq 0$ and $j_{1} \rightarrow \infty$, we obtain the Galerkin projection $P_{\infty, j_{2}}$ onto the space $V_{\infty, j_{2}}:=\left(I \otimes Q_{j_{2}}^{(2)}\right) \mathscr{H} \subset \mathscr{H}$. Analogously to (12), we find

$$
\left\|u-P_{\infty, j_{2}} u\right\|_{\mathscr{H}} \lesssim\left\|u-\left(I \otimes Q_{j_{2}}^{(2)}\right) u\right\|_{\mathscr{H}} \lesssim 2^{-j_{2} s_{2}}\|u\|_{\mathscr{H}_{\text {mix }}^{0, s_{2}}}
$$

for all $0<s_{2} \leq p_{2}$. 
With the help of the Galerkin projections, we can define

$$
\Delta_{j_{1}, j_{2}}^{P} u:=\left(P_{j_{1}, j_{2}}-P_{j_{1}-1, j_{2}}-P_{j_{1}, j_{2}-1}+P_{j_{1}-1, j_{2}-1}\right) u
$$

where we especially set $P_{j_{1},-1}:=0, P_{-1, j_{2}}:=0$, and $P_{-1,-1}:=0$. Then, the combination technique is expressed as the Boolean sum (cf. $[6,7,8]$ )

$$
\widehat{u}_{J}=\sum_{j_{1} \sigma+j_{2} / \sigma \leq J} \Delta_{j_{1}, j_{2}}^{P} u=u-\sum_{j_{1} \sigma+j_{2} / \sigma>J} \Delta_{j_{1}, j_{2}}^{P} u .
$$

Straightforward calculation shows

$$
\widehat{u}_{J}=\sum_{j_{1}=0}^{\lceil J / \sigma\rceil}\left(P_{j_{1},\left\lceil J \sigma-j_{1} \sigma^{2}\right\rceil}-P_{j_{1}-1,\left\lceil J \sigma-j_{1} \sigma^{2}\right\rceil}\right) u
$$

if $j_{1} \leq j_{2} \sigma^{2}$, and

$$
\widehat{u}_{J}=\sum_{j_{2}=0}^{\lceil\sigma \sigma\rceil}\left(P_{\left\lceil J / \sigma-j_{2} / \sigma^{2}\right\rceil, j_{2}}-P_{\left\lceil J / \sigma-j_{2} / \sigma^{2}\right\rceil, j_{2}-1}\right) u
$$

if $j_{1}>j_{2} \sigma^{2}$. A visualization of the formula (17) is found in Fig. 1 .

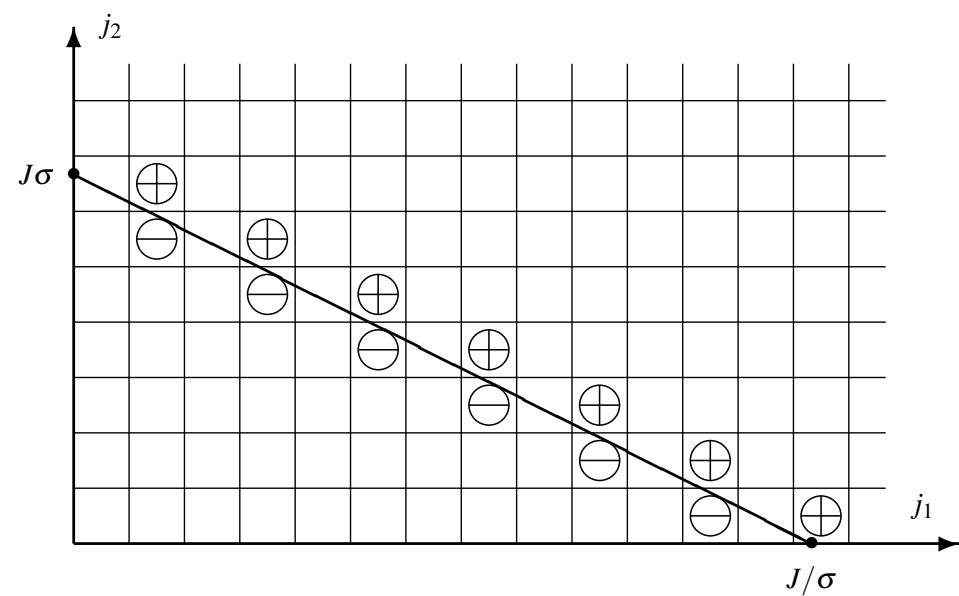

Fig. 1 The combination technique in $\widehat{V}_{J}^{\sigma}$ combines all the indicated solutions $P_{j_{1}, j_{2}} u$ with positive sign (" $\oplus$ ") and negative sign (" $\ominus$ ").

Our goal is now to show that the error $\left\|u-\widehat{u}_{J}\right\|_{\mathscr{H}}$ converges as good as the error of the true sparse tensor product Galerkin solution given in Corollary (1). 


\section{Proof of Convergence}

To prove the desired error estimate for the combination technique (16) and (17), respectively, we shall prove first the following two helpful lemmata.

Lemma 1. For all $0<s_{1} \leq p_{1}$ and $0<s_{2} \leq p_{2}$, it holds

$$
\begin{aligned}
\left\|\left(P_{j_{1}, j_{2}}-P_{j_{1}-1, j_{2}}\right) u\right\|_{\mathscr{H}} & \lesssim 2^{-j_{1} s_{1}}\|u\|_{\mathscr{H}_{\text {mix }}^{s_{1}, 0}} \\
\left\|\left(P_{j_{1}, j_{2}}-P_{j_{1}, j_{2}-1}\right) u\right\|_{\mathscr{H}} & \lesssim 2^{-j_{2} s_{2}}\|u\|_{\mathscr{H}_{\text {mix }}^{0, s_{2}}}
\end{aligned}
$$

provided that $u$ is sufficiently smooth and provided that the Galerkin projection satisfies

$$
\left\|P_{\infty, j_{2}} u\right\|_{\mathscr{H}_{\text {mix }}^{s_{1}, 0}} \lesssim\|u\|_{\mathscr{H}_{\text {mix }}^{s_{1}, 0}}, \quad\left\|P_{j_{1}, \infty} u\right\|_{\mathscr{H}_{\text {mix }}^{0, s_{2}}} \lesssim\|u\|_{\mathscr{H}_{\text {mix }}^{0, s_{2}}}
$$

Proof. We shall prove only the first estimate, the second one follows in complete analogy. To this end, we split

$$
\left\|\left(P_{j_{1}, j_{2}}-P_{j_{1}-1, j_{2}}\right) u\right\|_{\mathscr{H}} \leq\left\|\left(P_{j_{1}, j_{2}}-P_{\infty, j_{2}}\right) u\right\|_{\mathscr{H}}+\left\|\left(P_{\infty, j_{2}}-P_{j_{1}-1, j_{2}}\right) u\right\|_{\mathscr{H}} .
$$

Due to $V_{j_{1}-1, j_{2}}, V_{j_{1}, j_{2}} \subset V_{\infty, j_{2}}$, the associated Galerkin projections satisfy the identities $P_{j_{1}, j_{2}}=P_{j_{1}, j_{2}} P_{\infty, j_{2}}$ and $P_{j_{1}-1, j_{2}}=P_{j_{1}-1, j_{2}} P_{\infty, j_{2}}$. Hence, we obtain

$$
\left\|\left(P_{j_{1}, j_{2}}-P_{j_{1}-1, j_{2}}\right) u\right\|_{\mathscr{H}} \leq\left\|\left(P_{j_{1}, j_{2}}-I\right) P_{\infty, j_{2}} u\right\|_{\mathscr{H}}+\left\|\left(I-P_{j_{1}-1, j_{2}}\right) P_{\infty, j_{2}} u\right\|_{\mathscr{H}} .
$$

By employing now the fact that the Galerkin projections $P_{j_{1}-1, j_{2}} u$ and $P_{j_{1}, j_{2}} u$ are quasi-optimal, i.e., $\left\|\left(I-P_{j_{1}, j_{2}}\right) u\right\|_{\mathscr{H}} \lesssim\left\|\left(I-Q_{j_{1}}^{(1)} \otimes Q_{j_{2}}^{(2)}\right) u\right\|_{\mathscr{H}}$ and likewise for $P_{j_{1}-1, j_{2}} u$, we arrive at

$$
\begin{aligned}
& \left\|\left(P_{j_{1}, j_{2}}-P_{j_{1}-1, j_{2}}\right) u\right\|_{\mathscr{H}} \\
& \quad \lesssim\left\|\left(Q_{j_{1}}^{(1)} \otimes Q_{j_{2}}^{(2)}-I\right) P_{\infty, j_{2}} u\right\|_{\mathscr{H}}+\left\|\left(I-Q_{j_{1}-1}^{(1)} \otimes Q_{j_{2}}^{(2)}\right) P_{\infty, j_{2}} u\right\|_{\mathscr{H}} .
\end{aligned}
$$

The combination of $Q_{j_{1}}^{(1)} \otimes Q_{j_{2}}^{(2)}=\left(Q_{j_{1}}^{(1)} \otimes I\right)\left(I \otimes Q_{j_{2}}^{(2)}\right)$ and $\left(I \otimes Q_{j_{2}}^{(2)}\right) P_{\infty, j_{2}}=P_{\infty, j_{2}}$ yields the operator identity

$$
\left(Q_{j_{1}}^{(1)} \otimes Q_{j_{2}}^{(2)}\right) P_{\infty, j_{2}}=\left(Q_{j_{1}}^{(1)} \otimes I\right) P_{\infty, j_{2}}
$$

and likewise

$$
\left(Q_{j_{1}-1}^{(1)} \otimes Q_{j_{2}}^{(2)}\right) P_{\infty, j_{2}}=\left(Q_{j_{1}-1}^{(1)} \otimes I\right) P_{\infty, j_{2}} .
$$

Hence, we conclude

$$
\begin{aligned}
& \left\|\left(P_{j_{1}, j_{2}}-P_{j_{1}-1, j_{2}}\right) u\right\|_{\mathscr{H}} \\
& \quad \lesssim\left\|\left(\left(I-Q_{j_{1}}^{(1)}\right) \otimes I\right) P_{\infty, j_{2}} u\right\|_{\mathscr{H}}+\left\|\left(\left(I-Q_{j_{1}-1}^{(1)}\right) \otimes I\right) P_{\infty, j_{2}} u\right\|_{\mathscr{H}} \\
& \quad \lesssim 2^{-j_{1} s_{1}}\left\|P_{\infty, j_{2}} u\right\|_{\mathscr{H}_{\text {mix }}^{s_{1}, 0}} .
\end{aligned}
$$


Using the condition (18) implies finally the desired estimate.

Remark 1. Condition (18) holds if $A: \mathscr{H} \rightarrow \mathscr{H}^{\prime}$ is also continuous and bijective as a mapping $A: \mathscr{H}_{\text {mix }}^{s_{1}, 0} \rightarrow\left(\mathscr{H}^{\prime}\right)_{\text {mix }}^{s_{1}, 0}$ for all $0<s_{1} \leq p_{1}$ and also as as a mapping $A$ : $\mathscr{H}_{\text {mix }}^{0, s_{2}} \rightarrow\left(\mathscr{H}^{\prime}\right)_{\text {mix }}^{0, s_{2}}$ for all $0<s_{2} \leq p_{2}$, respectively. Then, in view of the continuity (5) of the projections $Q_{j_{1}}^{(1)}$ and $Q_{j_{2}}^{(2)}$, the Galerkin projections

$$
\begin{aligned}
P_{\infty, j_{2}} & =\left(\left(I \otimes Q_{j_{2}}^{(2)}\right) A\left(I \otimes Q_{j_{2}}^{(2)}\right)\right)^{-1}\left(I \otimes Q_{j_{2}}^{(2)}\right): \mathscr{H} \rightarrow V_{\infty, j_{2}} \subset \mathscr{H}, \\
P_{j_{1}, \infty} & =\left(\left(Q_{j_{1}}^{(1)} \otimes I\right) A\left(Q_{j_{1}}^{(1)} \otimes I\right)\right)^{-1}\left(Q_{j_{1}}^{(1)} \otimes I\right): \mathscr{H} \rightarrow V_{j_{1}, \infty} \subset \mathscr{H},
\end{aligned}
$$

are also continuous as mappings

$$
P_{\infty, j_{2}}: \mathscr{H}_{\text {mix }}^{s_{1}, 0} \rightarrow V_{\infty, j_{2}} \subset \mathscr{H}_{\text {mix }}^{s_{1}, 0}, \quad P_{j_{1}, \infty}: \mathscr{H}_{\text {mix }}^{0, s_{2}} \rightarrow V_{j_{1}, \infty} \subset \mathscr{H}_{\text {mix }}^{0, s_{2}},
$$

which implies (18).

Lemma 2. If $u \in \mathscr{H}_{\text {mix }}^{s_{1}, s_{2}}$, then it holds

$$
\left\|\left(\Delta_{j_{1}, j_{2}}^{P}-\Delta_{j_{1}, j_{2}}^{Q}\right) u\right\|_{\mathscr{H}} \lesssim 2^{-j_{1} s_{1}-j_{2} s_{2}}\|u\|_{\mathscr{H}_{m i x}^{s_{1}, s_{2}}}
$$

for all $0<s_{1} \leq p_{1}$ and $0<s_{2} \leq p_{2}$ where $\Delta_{j_{1}, j_{2}}^{Q}$ is given by (10) and $\Delta_{j_{1}, j_{2}}^{P}$ is given by (14), respectively.

Proof. Due to $P_{j_{1}, j_{2}}\left(Q_{j_{1}}^{(1)} \otimes Q_{j_{2}}^{(2)}\right)=Q_{j_{1}}^{(1)} \otimes Q_{j_{2}}^{(2)}$ for all $j_{1}, j_{2} \geq 0$, we obtain

$$
\begin{aligned}
\Delta_{j_{1}, j_{2}}^{P}-\Delta_{j_{1}, j_{2}}^{Q}= & P_{j_{1}, j_{2}}\left(I-Q_{j_{1}}^{(1)} \otimes Q_{j_{2}}^{(2)}\right)-P_{j_{1}-1, j_{2}}\left(I-Q_{j_{1}-1}^{(1)} \otimes Q_{j_{2}}^{(2)}\right) \\
& -P_{j_{1}, j_{2}-1}\left(I-Q_{j_{1}}^{(1)} \otimes Q_{j_{2}-1}^{(2)}\right)+P_{i-1, j-1}\left(I-Q_{j_{1}-1}^{(1)} \otimes Q_{j_{2}-1}^{(2)}\right) .
\end{aligned}
$$

We shall now make use of the identity

$$
\begin{aligned}
I-Q_{j_{1}}^{(1)} \otimes Q_{j_{2}}^{(2)} & =I \otimes I-Q_{j_{1}}^{(1)} \otimes Q_{j_{2}}^{(2)} \\
& =I \otimes\left(I-Q_{j_{2}}^{(2)}\right)+\left(I-Q_{j_{1}}^{(1)}\right) \otimes I-\left(I-Q_{j_{1}}^{(1)}\right) \otimes\left(I-Q_{j_{2}}^{(2)}\right) .
\end{aligned}
$$

Inserting this identity into (19) and reordering the terms yields 


$$
\begin{aligned}
\Delta_{j_{1}, j_{2}}^{P}-\Delta_{j_{1}, j_{2}}^{Q} & =\left(P_{j_{1}, j_{2}}-P_{j_{1}-1, j_{2}}\right)\left(I \otimes\left(I-Q_{j_{2}}^{(2)}\right)\right) \\
& -\left(P_{j_{1}, j_{2}-1}-P_{j_{1}-1, j_{2}-1}\right)\left(I \otimes\left(I-Q_{j_{2}-1}^{(2)}\right)\right) \\
& +\left(P_{j_{1}, j_{2}}-P_{j_{1}, j_{2}-1}\right)\left(\left(I-Q_{j_{1}}^{(1)}\right) \otimes I\right) \\
& -\left(P_{j_{1}-1, j_{2}}-P_{j_{1}-1, j_{2}-1}\right)\left(\left(I-Q_{j_{1}-1}^{(1)}\right) \otimes I\right) \\
& -P_{j_{1}, j_{2}}\left(\left(I-Q_{j_{1}}^{(1)}\right) \otimes\left(I-Q_{j_{2}}^{(2)}\right)\right) \\
& +P_{j_{1}-1, j_{2}}\left(\left(I-Q_{j_{1}-1}^{(1)}\right) \otimes\left(I-Q_{j_{2}}^{(2)}\right)\right) \\
& +P_{j_{1}, j_{2}-1}\left(\left(I-Q_{j_{1}}^{(1)}\right) \otimes\left(I-Q_{j_{2}-1}^{(2)}\right)\right) \\
& -P_{j_{1}-1, j_{2}-1}\left(\left(I-Q_{j_{1}-1}^{(1)}\right) \otimes\left(I-Q_{j_{2}-1}^{(2)}\right)\right) .
\end{aligned}
$$

The combination of the error estimates

$$
\begin{aligned}
\left\|\left(P_{j_{1}, j_{2}}-P_{j_{1}-1, j_{2}}\right) u\right\|_{\mathscr{H}} & \lesssim 2^{-j_{1} s_{1}}\|u\|_{\mathscr{H}_{\operatorname{mix}}^{s_{1}, 0}}, \\
\left\|\left(P_{j_{1}, j_{2}}-P_{j_{1}, j_{2}-1}\right) u\right\|_{\mathscr{H}} & \lesssim 2^{-j_{2} s_{2}}\|u\|_{\mathscr{H}_{\operatorname{mix}}^{0, s_{2}}},
\end{aligned}
$$

cf. Lemma 1, and

$$
\begin{gathered}
\left\|\left(I \otimes\left(I-Q_{j_{2}}^{(2)}\right)\right) u\right\|_{\mathscr{H}_{\text {mix }}^{s_{1}, 0}} \lesssim 2^{-j_{2} s_{2}}\|u\|_{\mathscr{H}_{\text {mix }}^{s_{1}, s_{2}}}, \\
\left\|\left(\left(I-Q_{j_{1}}^{(1)}\right) \otimes I\right) u\right\|_{\mathscr{H}_{\text {mix }}^{0, s_{2}}} \lesssim 2^{-j_{1} s_{1}}\|u\|_{\mathscr{H}_{\text {mix }}^{s_{1}, s_{2}}},
\end{gathered}
$$

leads to

$$
\begin{gathered}
\left\|\left(P_{j_{1}, j_{2}}-P_{j_{1}-1, j_{2}}\right)\left(I \otimes\left(I-Q_{j_{2}}^{(2)}\right)\right) u\right\|_{\mathscr{H}} \lesssim 2^{-j_{1} s_{1}-j_{2} s_{2}}\|u\|_{\mathscr{H}_{\text {mix }}^{s_{1}, s_{2}}}, \\
\left\|\left(P_{j_{1}, j_{2}}-P_{j_{1}, j_{2}-1}\right)\left(\left(I-Q_{j_{1}}^{(1)}\right) \otimes I\right) u\right\|_{\mathscr{H}} \lesssim 2^{-j_{1} s_{1}-j_{2} s_{2}}\|u\|_{\mathscr{H}_{\text {mix }}^{s_{1}, s_{2}}} .
\end{gathered}
$$

Similarly, from the continuity

$$
\left\|P_{j_{1}, j_{2}} u\right\|_{\mathscr{H}} \lesssim\|u\|_{\mathscr{H}}
$$

and

$$
\left\|\left(\left(I-Q_{j_{1}}^{(1)}\right) \otimes\left(I-Q_{j_{2}}^{(2)}\right)\right) u\right\|_{\mathscr{H}} \lesssim 2^{-j_{1} s_{1}-j_{2} s_{2}}\|u\|_{\mathscr{H}_{\text {mix }}^{s_{1}, s_{2}}},
$$

we deduce

$$
\left\|P_{j_{1}, j_{2}}\left(\left(I-Q_{j_{1}}^{(1)}\right) \otimes\left(I-Q_{j_{2}}^{(2)}\right)\right) u\right\|_{\mathscr{H}} \lesssim 2^{-j_{1} s_{1}-j_{2} s_{2}}\|u\|_{\mathscr{H}_{\text {mix }}^{s_{1}, s_{2}}} .
$$

With (20) and (21) at hand, we can estimate each of the eight different terms which yields the desired error estimate 


$$
\left\|\left(\Delta_{j_{1}, j_{2}}^{P}-\Delta_{j_{1}, j_{2}}^{Q}\right) u\right\|_{\mathscr{H}} \lesssim 2^{-j_{1} s_{1}-j_{2} s_{2}}\|u\|_{\mathscr{H}_{\text {mix }}^{s_{1}, s_{2}}} .
$$

Now, we arrive at our main result which proves optimal convergence rates.

Theorem 2. The solution (16) and (17), respectively, of the combination technique satisfies the error estimate

$$
\left\|u-\widehat{u}_{J}\right\|_{\mathscr{H}} \lesssim \begin{cases}2^{-J \min \left\{s_{1} / \sigma, s_{2} \sigma\right\}}\|u\|_{\mathscr{H}_{\text {mix }}^{s_{1}, s_{2}},} & \text { if } s_{1} / \sigma \neq s_{2} \sigma, \\ 2^{-J s_{1} / \sigma \sqrt{J}\|u\|_{\mathscr{H}}^{s_{1 i x}, s_{2}},} & \text { if } s_{1} / \sigma=s_{2} \sigma,\end{cases}
$$

for all $0<s_{1} \leq p_{1}$ and $0<s_{2} \leq p_{2}$ provided that $u \in H_{\text {mix }}^{s_{1}, s_{2}}\left(\Omega_{1} \times \Omega_{2}\right)$.

Proof. In view of (15), we have

$$
\left\|u-\widehat{u}_{J}\right\|_{\mathscr{H}}^{2}=\left\|\sum_{j_{1} \sigma+j_{2} / \sigma>J} \Delta_{j_{1}, j_{2}}^{P} u\right\|_{\mathscr{H}}^{2} .
$$

The Galerkin orthogonality implies the relation

$$
\left\|\sum_{j_{1} \sigma+j_{2} / \sigma>J} \Delta_{j_{1}, j_{2}}^{P} u\right\|_{\mathscr{H}}^{2} \sim \sum_{j_{1} \sigma+j_{2} / \sigma>J}\left\|\Delta_{j_{1}, j_{2}}^{P} u\right\|_{\mathscr{H}}^{2} .
$$

Thus, we arrive at

$$
\left\|u-\widehat{u}_{J}\right\|_{\mathscr{H}}^{2} \lesssim \sum_{j_{1} \sigma+j_{2} / \sigma>J}\left\|\Delta_{j_{1}, j_{2}}^{Q} u\right\|_{\mathscr{H}}^{2}+\sum_{j_{1} \sigma+j_{2} / \sigma>J}\left\|\left(\Delta_{j_{1}, j_{2}}^{P}-\Delta_{j_{1}, j_{2}}^{Q}\right) u\right\|_{\mathscr{H}}^{2} .
$$

We bound the first sum on the right hand side in complete analogy to [10] from above by

$$
\begin{aligned}
\sum_{j_{1} \sigma+j_{2} / \sigma>J}\left\|\Delta_{j_{1}, j_{2}}^{Q} u\right\|_{\mathscr{H}}^{2} & \lesssim \sum_{j_{1} \sigma+j_{2} / \sigma>J} 2^{-2 j_{1} s_{1}-2 j_{2} s_{2}}\|u\|_{\mathscr{H}_{\text {mix }}^{s_{1}, s_{2}}}^{2} \\
& \lesssim \begin{cases}2^{-2 J \min \left\{s_{1} / \sigma, s_{2} \sigma\right\}}\|u\|_{\mathscr{H}_{\text {mix }}^{s_{1}, s_{2}}}^{2}, & \text { if } s_{1} / \sigma \neq s_{2} \sigma, \\
2^{-2 J s_{1} / \sigma} J\|u\|_{\mathscr{H}_{\text {mix }}^{s_{1}}}^{2}, & \text { if } s_{1} / \sigma=s_{2} \sigma .\end{cases}
\end{aligned}
$$

Likewise, with the help of Lemma 2, the second sum on the right hand side is bounded from above by

$$
\begin{aligned}
& \sum_{j_{1} \sigma+j_{2} / \sigma>J}\left\|\left(\Delta_{j_{1}, j_{2}}^{P}-\Delta_{j_{1}, j_{2}}^{Q}\right) u\right\|_{\mathscr{H}}^{2} \lesssim \sum_{j_{1} \sigma+j_{2} / \sigma>J} 2^{-2 j_{1} s_{1}-2 j_{2} s_{2}}\|u\|_{\mathscr{H}_{\text {mix }}^{s_{1}, s_{2}}}^{2} \\
& \lesssim \begin{cases}2^{-2 J \min \left\{s_{1} / \sigma, s_{2} \sigma\right\}}\|u\|_{\mathscr{H}_{\text {mix }}^{s_{1}, s_{2}}}^{2}, & \text { if } s_{1} / \sigma \neq s_{2} \sigma, \\
2^{-2 J s_{1} / \sigma} J\|u\|_{\mathscr{H}_{\text {mix }}^{s_{1}, s_{2}}}^{2}, & \text { if } s_{1} / \sigma=s_{2} \sigma,\end{cases}
\end{aligned}
$$


which, altogether, yields the desired error estimate.

\section{Numerical results}

We now validate our theoretical findings by numerical experiments. Specifically, we will apply the combination technique for the three examples which were mentioned in Section 2. To this end, we consider the most simple case and choose $\Omega_{1}=\Omega_{2}=$ $(0,1)$, i.e., $n_{1}=n_{2}=1$. The ansatz spaces $V_{j}^{(1)}$ and $V_{j}^{(2)}$ consist of continuous, piecewise linear ansatz functions on an equidistant subdivision of the interval $(0,1)$ into $2^{j}$ subintervals. This yields the polynomial exactnesses $r_{1}=r_{2}=2$. For the sake of notational convenience, we set $\square=(0,1) \times(0,1)$.

Example 1. First, we solve the variational problem

$$
\text { find } u \in L^{2}(\square) \text { such that } a(u, v)=\ell(v) \text { for all } v \in L^{2}(\square)
$$

where

$$
a(u, v)=\int_{\square} \alpha(x, y) u(x, y) v(x, y) \mathrm{d}(x, y)
$$

and

$$
\ell(v)=\int_{\square} f(x, y) v(x, y) \mathrm{d}(x, y) .
$$

The underlying operator $A$ is the multiplication operator

$$
(A u)(x, y)=\alpha(x, y) u(x, y)
$$

which is of the order 0 . Hence, we have the energy space $\mathscr{H}=L^{2}(\square)$ and the related spaces of assumed stronger regularity are $\mathscr{H}_{\text {mix }}^{s_{1}, s_{2}}=H_{\text {mix }}^{s_{1}, s_{2}}(\square)$. If the multiplier $\alpha(x, y)$ is a smooth function, then $A$ arbitrarily shifts through the Sobolev scales which implies the condition (18) due to Remark 1.

Let the solution $u$ be a smooth function such that $u \in \mathscr{H}_{\text {mix }}^{s_{1}, s_{2}}$ for given $s_{1}, s_{2} \geq 0$, which holds if the right hand side $f$ is sufficiently regular. Then, the best possible approximation rate for the present discretization with piecewise linear ansatz functions is obtained for $s_{1}=r_{1}=2$ and $s_{2}=r_{2}=2$, i.e., for $\mathscr{H}_{\text {mix }}^{s_{1}, s_{2}}=H_{\text {mix }}^{2,2}(\square)$. Thus, the regular sparse tensor product space

$$
\widehat{V}_{J}^{1}=\bigoplus_{j_{1}+j_{2} \leq J} W_{j_{1}}^{(1)} \otimes W_{j_{2}}^{(2)}=\sum_{j_{1}+j_{2}=J} V_{j_{1}}^{(1)} \otimes V_{j_{2}}^{(2)} .
$$

(cf. (8)) is optimal for the discretization, see [10] for a detailed derivation. In particular, with Theorem 2, the combination technique yields the error estimate

$$
\left\|u-\widehat{u}_{J}\right\|_{L^{2}(\square)} \lesssim 4^{-J} \sqrt{J}\|u\|_{H_{m i x}^{2,2}(\square)} .
$$

For our numerical tests, we choose 


$$
\alpha(x, y)=1+(x+y)^{2}, \quad f(x, y)=\alpha(x, y) u(x, y), \quad u(x, y)=\sin (\pi x) \sin (\pi y) .
$$

The resulting convergence history is plotted as the red curve in Fig. 2. As can be seen there, the convergence rate $4^{-J} \sqrt{J}$, indicated by the dashed red line, is indeed obtained in the numerical experiments.

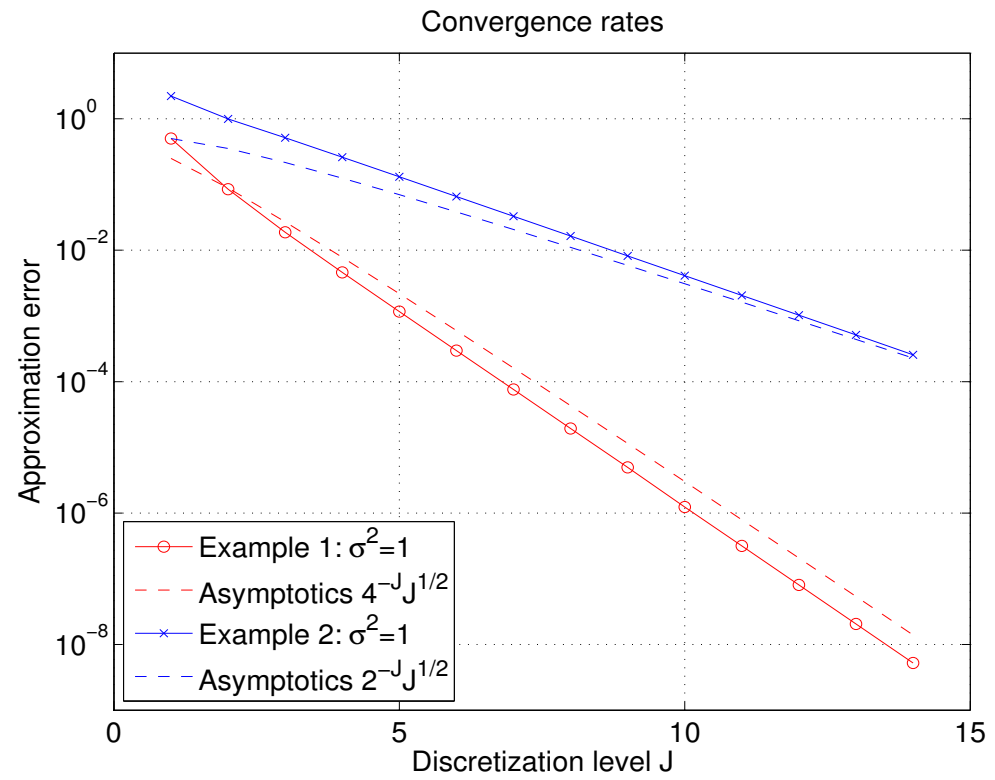

Fig. 2 Convergence rates in case of the first and second example.

Example 2. This example concerns the stationary heat conduction in the domain $\square$. In its weak form, it is given by the variational problem

$$
\text { find } u \in H_{0}^{1}(\square) \text { such that } a(u, v)=\ell(v) \text { for all } v \in H_{0}^{1}(\square)
$$

where

$$
a(u, v)=\int_{\square} \alpha(x, y)\left\{\frac{\partial u}{\partial x}(x, y) \frac{\partial v}{\partial x}(x, y)+\frac{\partial u}{\partial y}(x, y) \frac{\partial v}{\partial y}(x, y)\right\} \mathrm{d}(x, y)
$$

and $\ell(v)$ as in (22). The underlying operator $A$ is the elliptic second order differential operator

$$
(A u)(x, y)=-\operatorname{div}_{(x, y)}\left(\alpha(x, y) \nabla_{(x, y)} u(x, y)\right)
$$


and maps the energy space $\mathscr{H}=H_{0}^{1}(\square)$ bijectively onto its dual $\mathscr{H}^{\prime}=H^{-1}(\square)$. Recall that now the spaces of assumed stronger regularity are $\mathscr{H}_{\text {mix }}^{s_{1}, s_{2}}=H_{0}^{1}(\square) \cap$ $H_{\text {mix }}^{s_{1}+1, s_{2}}(\square) \cap H_{\text {mix }}^{s_{1}, s_{2}+1}(\square)$.

Since the domain $\square$ is convex, the second order boundary value problem under consideration is $H^{2}$-regular, which implies that $A: H_{0}^{1}(\square) \cap H^{2}(\square) \rightarrow L^{2}(\square)$ is also bijective. By interpolation arguments, we thus find that $A: \mathscr{H}_{\text {mix }}^{1,0} \rightarrow\left(\mathscr{H}^{\prime}\right)_{\text {mix }}^{1,0}$ is continuous and bijective since

$$
L^{2}(\square) \subset\left(\mathscr{H}^{\prime}\right)_{\text {mix }}^{1,0} \subset H^{-1}(\square) \quad \text { and } \quad H_{0}^{1}(\square) \cap H^{2}(\square) \subset \mathscr{H}_{\text {mix }}^{1,0} \subset H_{0}^{1}(\square) .
$$

Likewise, $A: \mathscr{H}_{\text {mix }}^{0,1} \rightarrow\left(\mathscr{H}^{\prime}\right)_{\text {mix }}^{0,1}$ is continuous and bijective. Hence, the condition (18) holds again due to Remark 1 and Lemma 1 applies.

Again, the regular sparse tensor product space (23) is optimal for the present discretization with piecewise linear ansatz functions. Consequently, Theorem $2 \mathrm{implies}$ as the best possible convergence estimate

$$
\left\|u-\widehat{u}_{J}\right\|_{H^{1}(\square)} \lesssim 2^{-J} \sqrt{J}\|u\|_{H_{\text {mix }}^{2,1}(\square) \cap H_{\text {mix }}^{1,2}(\square)}
$$

provided that $u \in H_{\text {mix }}^{2,1}(\square) \cap H_{\text {mix }}^{1,2}(\square)$. Here, we exploited that $\mathscr{H}_{\text {mix }}^{1,1}=H_{0}^{1}(\square) \cap$ $H_{\text {mix }}^{2,1}(\square) \cap H_{\text {mix }}^{1,2}(\square)$. Nevertheless, in general, we only have $u \in H^{2}(\square) \not \subset H_{\text {mix }}^{2,1}(\square) \cap$ $H_{\text {mix }}^{1,2}(\square)$. Thus, due to $H_{\text {mix }}^{3 / 2,1 / 2}(\square) \cap H_{\text {mix }}^{1 / 2,3 / 2}(\square) \subset H^{2}(\square)$, one can only expect the reduced convergence rate

$$
\left\|u-\widehat{u}_{J}\right\|_{H^{1}(\square)} \lesssim 2^{-J / 2} \sqrt{J}\|u\|_{H^{2}(\square)} .
$$

In our particular numerical computations, we use

$$
\begin{gathered}
\alpha(x, y)=1+(x+y)^{2}, \quad u(x, y)=\sin (\pi x) \sin (\pi y), \\
f(x, y)=\frac{\partial a}{\partial x}(x, y) \frac{\partial u}{\partial x}(x, y)+\frac{\partial a}{\partial y}(x, y) \frac{\partial u}{\partial y}(x, y)-\alpha(x, y) \Delta u(x, y) .
\end{gathered}
$$

Therefore, due to $u \in H_{\text {mix }}^{2,1}(\square) \cap H_{\text {mix }}^{1,2}(\square)$, we should observe the convergence rate $2^{-J} \sqrt{J}$. The computational approximation errors are plotted as the blue graph in Figure 2 . The dashed blue line corresponds to $2^{-J} \sqrt{J}$ and clearly validates the predicted convergence rate. We even observe the slighty better rate $2^{-J}$ which can be explained by the fact that the solution $u$ is even in $H_{\text {mix }}^{2,2}(\square)$, see [2] for the details.

Example 3. We shall finally consider the variational problem

find $u \in L^{2}(0,1) \otimes H_{0}^{1}(0,1)$ such that $a(u, v)=\ell(v)$ for all $v \in L^{2}(0,1) \otimes H_{0}^{1}(0,1)$

where

$$
a(u, v)=\int_{\square} \alpha(x, y) \frac{\partial u}{\partial y}(x, y) \frac{\partial v}{\partial y}(x, y) \mathrm{d}(x, y)
$$


and $\ell(v)$ is again given as in (22). The underlying operator $A$ is the elliptic differential operator

$$
(A u)(x, y)=-\frac{\partial}{\partial y}\left(\alpha(x, y) \frac{\partial}{\partial y} u(x, y)\right) .
$$

Its energy space is $\mathscr{H}=L^{2}(0,1) \otimes H_{0}^{1}(0,1) \subset H_{\text {mix }}^{0,1}(\square)$ with dual $\mathscr{H}^{\prime}=L^{2}(0,1) \otimes$ $H^{-1}(0,1)$. Here, the spaces of assumed stronger regularity coincide with $\mathscr{H}_{\text {mix }}^{s_{1}, s_{2}}=$ $\left(L^{2}(0,1) \otimes H_{0}^{1}(0,1)\right) \cap H_{\text {mix }}^{s_{1}, s_{2}+1}(\square)$.

The operator $A$ shifts as a operator $\mathscr{H}_{\text {mix }}^{s_{1}, s_{2}+1} \rightarrow\left(\mathscr{H}^{\prime}\right)_{\text {mix }}^{s_{1}, s_{2}+1}$ for arbitrary $s_{1}, s_{2} \geq$ 0 provided that the coeffcient $\alpha$ is smooth enough. Thus, Theorem 2 holds and predicts the best possible convergence estimate for our underlying discretization with piecewise linear ansatz functions if $u$ lies in the space $H_{m i x}^{2,2}(\square)$.

According to the theory presented in [10], the optimal cost complexity with respect to the generalized sparse tensor product spaces $\widehat{V}_{J}^{\sigma}$ is obtained for the choice

$$
\sigma \in\left[\sqrt{\frac{n_{1}}{n_{2}}}, \sqrt{\frac{r_{1}}{r_{2}-1}}\right]=[1, \sqrt{2}] .
$$

In order to be able to compare the convergence rates instead of the cost complexities for different choices of $\sigma$, we have to consider the generalized sparse tensor product spaces $\widehat{V}_{\bar{J}}^{\sigma}$, where $\bar{J}:=\sigma J$. Then, for all the above choices of $\sigma$, we essentially expect the convergence rate

$$
\left\|u-\widehat{u}_{\bar{J}}\right\|_{H_{\text {mix }}^{0,1}(\square)} \lesssim 2^{-\bar{J} / \sigma}\|u\|_{H_{\text {mix }}^{2,2}(\square)} \sim 2^{-J}\|u\|_{H_{\text {mix }}^{2,2}(\square)}
$$

while the degrees of freedom of $\widehat{V}_{\bar{J}}^{\sigma}$ essentially scale like $2^{\bar{J} / \sigma} \sim 2^{J}$. This setting is employed in our numerical tests, where we further set

$$
\begin{gathered}
\alpha(x, y)=1+(x+y)^{2}, \quad u(x, y)=\sin (\pi x) \sin (\pi y), \\
f(x, y)=\frac{\partial a}{\partial y}(x, y) \frac{\partial u}{\partial y}(x, y)-\alpha(x, y) \frac{\partial^{2} u}{\partial y^{2}}(x, y) .
\end{gathered}
$$

We apply the combination technique for the particular choices

- $\sigma=1$, which yields an equilibration of the unknowns in all the extremal tensor product spaces $W_{j_{1}}^{(1)} \otimes W_{\bar{J}-j_{1} \sigma^{2}}^{(2)}$,

- $\sigma=\sqrt{2}$, which yields an equilibration of the approximation in all the extremal tensor product spaces $W_{j_{1}}^{(1)} \otimes W_{J_{-} j_{1} \sigma^{2}}^{(2)}$, and

- $\sigma=\sqrt{3 / 2}$, which results in an equilibrated cost-benefit rate, see $[1,10]$ for the details.

The computed approximation errors are found in Fig. 3, where the red curve corresponds to $\sigma=1$, the black curve corresponds to $\sigma=\sqrt{2}$, and the blue curve corresponds to $\sigma=\sqrt{3 / 2}$. In the cases $\sigma=1$ and $\sigma=\sqrt{2}$, we achieve the predicted convergence rate $2^{-J}$ which is indicated by the dashed black line. In the case 


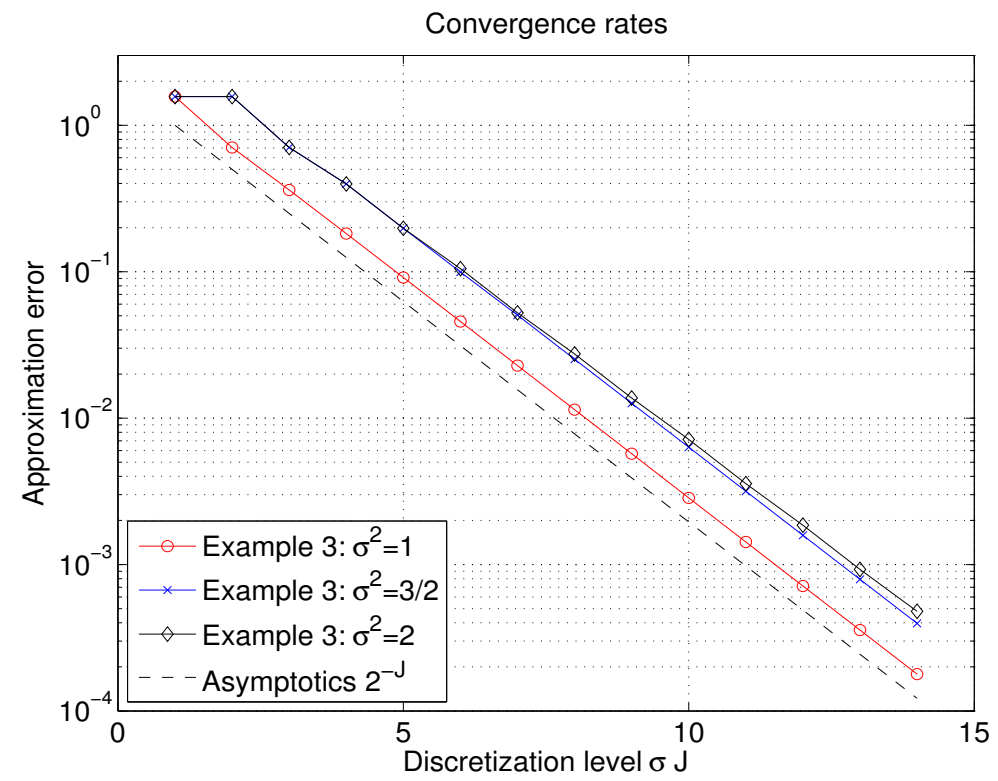

Fig. 3 Convergence rates in case of the third example.

$\sigma=\sqrt{2}$ the predicted convergence rate is only $2^{-J} \sqrt{J}$ which is also confirmed by Fig. 3 .

\section{Conclusion}

In the present paper, we proved the convergence of the combination technique in a rather general set-up. Especially, we considered the combination technique in generalized sparse tensor product spaces. We restricted ourselves here to the case of two-fold tensor product domains. Nevertheless, all our results can straightforwardly be extended to the case of generalized $L$-fold sparse tensor product spaces by applying the techniques from [11] and [25]. Then, of course, the constants hidden by the " $\sim$ " -notation will depend on the given dimension $L$.

\section{References}

1. H-J. Bungartz and M. Griebel. Sparse grids. Acta Numer., 13:147-269, 2004.

2. H-J. Bungartz and M. Griebel. A note on the complexity of solving Poisson's equation for spaces of bounded mixed derivatives. J. Complexity 15(2):167-199, 1999. 
3. H. Bungartz, M. Griebel, D. Röschke, and C. Zenger. A proof of convergence for the combination technique for the Laplace equation using tools of symbolic computation. Math. Comput. Simulation, 42(4-6):595-605, 1996.

4. D. Cioranescu, A. Damlamian, and G. Griso. The periodic unfolding method in homogenization. SIAM J. Math. Anal., 40(4):1585-1620, 2008.

5. W. Dahmen. Wavelet and multiscale methods for operator equations. Acta Numer., 6:55-228, 1997.

6. F.-J. Delvos. $d$-variate Boolean interpolation. J. Approx. Theory 34(2):99-114, 1982.

7. F.-J. Delvos. Boolean methods for double integration. Math. Comp. 55(192):683-692, 1990.

8. F.-J. Delvos and W. Schempp. Boolean methods in interpolation and approximation. Volume 230 of Pitman Research Notes in Mathematics Series, Longman Scientific \& Technical, Harlow, 1989.

9. M. Griebel. Multilevelmethoden als Iterationsverfahren über Erzeugendensystemen. Teubner Skripten zur Numerik. B.G. Teubner, Stuttgart, 1994.

10. M. Griebel and H. Harbrecht. On the construction of sparse tensor product spaces. Math. Comput., 82(282):975-994, 2013.

11. M. Griebel and H. Harbrecht. A note on the construction of $L$-fold sparse tensor product spaces. Constr. Approx., 38(2):235-251, 2013.

12. M. Griebel and P. Oswald. On additive Schwarz preconditioners for sparse grid discretizations. Numer. Math., 66:449-463, 1994.

13. M. Griebel, P. Oswald, and T. Schiekofer. Sparse grids for boundary integral equations. Numer. Math., 83(2):279-312, 1999.

14. M. Griebel, M. Schneider, and C. Zenger. A combination technique for the solution of sparse grid problems. In: Iterative Methods in Linear Algebra, edited by P. de Groen and R. Beauwens, pp. 263-281. IMACS, Elsevier, North Holland, 1992.

15. H. Harbrecht. A finite element method for elliptic problems with stochastic input data. Appl. Numer. Math., 60(3):227-244, 2010.

16. H. Harbrecht, M. Peters, and M. Siebenmorgen. Combination technique based $k$-th moment analysis of elliptic problems with random diffusion. J. Comput. Phys., 252:128-141, 2013.

17. H. Harbrecht, R. Schneider, and C. Schwab. Sparse second moment analysis for elliptic problems in stochastic domains. Numer. Math., 109(3):385-414, 2008.

18. H. Harbrecht, R. Schneider, and C. Schwab. Multilevel frames for sparse tensor product spaces. Numer. Math., 110(2):199-220, 2008.

19. M. Hegland, J. Garcke, and V. Challis. The combination technique and some generalisations. Linear Alg. Appl., 420(2-3):249-275, 2007.

20. V.H. Hoang and C. Schwab. High-dimensional finite elements for elliptic problems with multiple scales. Multiscale Model. Simul., 3(1):168-194, 2004/05.

21. C. Pflaum. Convergence of the combination technique for second-order elliptic differential equation. SIAM J. Numer. Anal., 34:2431-2455,1997.

22. C. Pflaum and A. Zhou. Error analysis of the combination technique. Numer. Math., 84:327$350,1999$.

23. C. Reisinger. Analysis of linear difference schemes in the sparse grid combination technique. IMA J. Numer. Analysis, 33(2):544-581, 2013.

24. C. Schwab and R.-A. Todor. Sparse finite elements for elliptic problems with stochastic loading. Numer. Math., 95(4):707-734, 2003.

25. Y. Xu and A. Zhou. Fast Boolean approximation methods for solving integral equations in high dimensions. J. Integral Equations Appl., 16(1):83-110, 2004.

26. C. Zenger. Sparse grids. In: Parallel algorithms for partial differential equations. Proc. 6th GAMM-Semin., Kiel/Germany 1990, edited by W. Hackbusch, Notes Numer. Fluid Mech. 31, pp. 241-251. Vieweg, Braunschweig, 1991. 